\title{
Measurements of the Higgs production cross section in the $H \rightarrow \tau \tau$ decay channel with the ATLAS experiment
}

\author{
Alessia Murrone* on behalf of the ATLAS collaboration \\ Universitá degli Studi di Milano and INFN Milano, (Italy) \\ E-mail: alessia.murrone@cern.ch
}

\begin{abstract}
Measurements of the Higgs production cross section in the $H \rightarrow \tau \tau$ decay channel are presented. The analysis has been performed using $36.1 \mathrm{fb}^{-1}$ of data collected by the ATLAS experiment at $\sqrt{s}=13 \mathrm{TeV}$ proton-proton collisions at the Large Hadron Collider. The observed (expected) significance of the $H \rightarrow \tau \tau$ signal excess over the expected background amounts to 4.4 (4.1) standard deviations. This result, combined with the data taken at $\sqrt{s}=7$ and $8 \mathrm{TeV}$, leads to an observed (expected) significance of 6.4 (5.4) and constitutes the first ATLAS observation of $H \rightarrow \tau \tau$. The measured total cross section of $H \rightarrow \tau \tau$, using the data collected at $\sqrt{s}=13 \mathrm{TeV}$, is $3.77_{-0.59}^{+0.60}$ (stat.) ${ }_{-0.74}^{+0.87}$ (syst.) pb, assuming the relative contributions of the Higgs production processes as predicted by the Standard Model. In addition, total cross sections for the vector boson fusion and gluon-gluon fusion processes have been measured separately and similar results are reported based on the simplified template cross section framework. All the measurements are in agreement with the Standard Model predictions.
\end{abstract}

European Physical Society Conference on High Energy Physics - EPS-HEP2019 -

10-17 July, 2019

Ghent, Belgium

${ }^{*}$ Speaker. 


\section{Introduction}

In 2012 the ATLAS and CMS experiments discovered a particle consistent with the Standard Model (SM) Higgs boson [1, 2]. After the Higgs boson discovery, the studies have been focused on measuring its properties such as coupling strengths, spin and CP quantum numbers. Several measurements have been performed during Run 1 of the LHC at a center of mass energy of $\sqrt{s}=7$ and $8 \mathrm{TeV}$ and they have not shown any deviation from SM expectations. Among Higgs couplings to fermions, only the ones to third generation fermions are currently accessible at the LHC. The $H \rightarrow \tau \tau$ decay channel is of considerable importance since it allows the Higgs Yukawa coupling to leptons to be directly measured ${ }^{1}$. The observation of the $H \rightarrow \tau \tau$ decay has already been established by the ATLAS and CMS combined measurement using Run 1 data, with a significance of 5.5 $\sigma$ [3]. Nevertheless the $H \rightarrow \tau \tau$ channel can benefit from the higher Run 2 statistics in order to reach larger precision results.

The analysis [4] presented in this article has been performed using the 2015 and 2016 data recorded by the ATLAS detector [5] at the LHC, corresponding to an integrated luminosity of $36.1 \mathrm{fb}^{-1}$. All combinations of possible decay modes have been exploited in the analysis: both leptonic $(\tau \rightarrow l v \bar{v}$ with $l=e, \mu$ ) and hadronic ( $\tau \rightarrow$ hadrons $v$ ) $\tau$ decays. The three decay channels will henceforth be referred to as $\tau_{\text {lep }} \tau_{\text {lep }}, \tau_{\text {lep }} \tau_{\text {had }}$ and $\tau_{\text {had }} \tau_{\text {had }}$.

\section{Event selection}

The analysis aims at measuring the Higgs boson production cross section exploiting signalsensitive event topologies. Therefore, two kinds of signal region (SR) are defined: one region called "VBF", which targets events produced through vector boson fusion (VBF). and a region called "boosted". which targets events produced through gluon-gluon fusion (ggF), with an additional recoiling jet. These two kinds of SRs are defined applying some kinematic requirements. The VBF is characterized by two high- $p_{\mathrm{T}}$ jets, which are required to have a large pseudorapidity separation $\left(\left|\Delta \eta_{j j}\right|>3\right)$ and large invariant mass $\left(m_{j j}>400 \mathrm{GeV}\right)$. The boosted category contains instead events that fail the VBF selection and are characterized by a high- $p_{\mathrm{T}}$ Higgs boson $\left(p_{\mathrm{T}}^{H}>100 \mathrm{GeV}\right)$. In the analysis the SR are further split into subregions in order to increase the sensitivity, for a total of thirteen SR. Figure 1 shows the $m_{\tau \tau}^{\mathrm{MMC}}$ distributions for the sum of all VBF (a) and the sum of all boosted (b) regions, where the $m_{\tau \tau}^{\mathrm{MMC}}$ is the Higgs invariant mass obtained with the Missing Mass Calculator (MMC) [6] algorithm.

In addition, six control regions (CRs) are used to constrain the $Z \rightarrow l l$ and the top-quark backgrounds and one validation region (VR) is used to verify the correct modelling of the $Z \rightarrow \tau \tau$ background, as will be illustrated in Section 3 .

\section{Background estimation}

The different final states of the three decay channels imply different background compositions and also need different strategies for background estimation.

The Drell-Yan process $Z / \gamma^{*} \rightarrow \tau \tau$ constitutes the main irreducible background. In addition, the

\footnotetext{
${ }^{1}$ Limits have been set to the $H \rightarrow \mu \mu$ but there is not an evidence yet.
} 


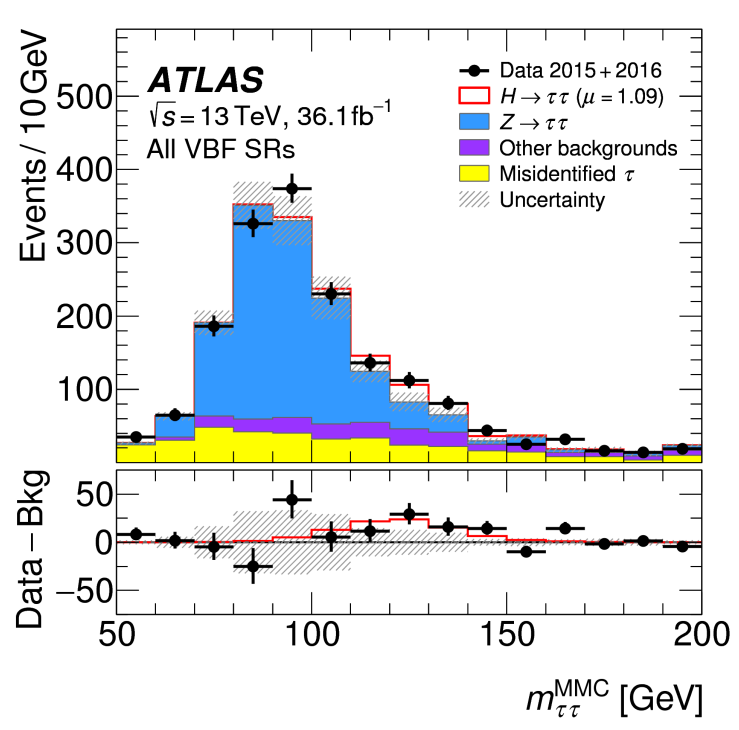

(a)

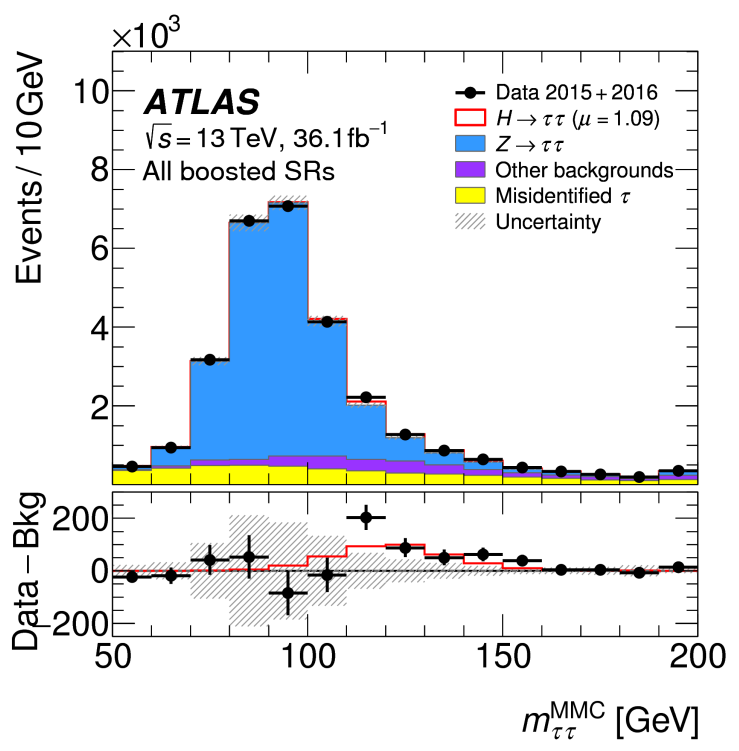

(b)

Figure 1: $m_{\tau \tau}^{\mathrm{MMC}}$ distributions for the sum of all VBF (a) and all boosted (b) signal regions. The bottom panel shows the difference between observed data and expected background [4].

separation between $Z \rightarrow \tau \tau$ and $H \rightarrow \tau \tau$ is limited by the $m_{\tau \tau}^{\mathrm{MMC}}$ resolution. This background is estimated using simulations, namely using the SHERPA [7] Monte Carlo generator at Next-ToLeading Order (NLO). The normalization of this major background is directly retrieved from the fit to data and no specific control region is defined. Therefore, in order to verify the correctness of the $Z \rightarrow \tau \tau$ background modeling, a validation region is used instead. Since it is difficult to select a pure $Z \rightarrow \tau \tau$ sample, this region is built using a selection based on the $\tau_{\text {lep }} \tau_{\text {lep }}$ SR and using $Z \rightarrow l l$ events.

Another significant background arises from hadronic jets which are misidentified as $\tau_{\text {had }}$ or as electrons or muons. In order to estimate this background different data-driven techniques are used in the three decay channels. The main sources of this background are QCD jets and $W / Z$ produced in association with jets. In the case of $\tau_{\text {lep }} \tau_{\text {lep }}$ channel, templates are built in dedicated control regions and the normalization is retrieved from extrapolation to the signal region. The $\tau_{\text {lep }} \tau_{\text {had }}$ channel uses a "fake-factor" method, where the fraction of misidentified events is derived in dedicated control regions and propagated to the signal region. The normalisation is derived from control region extrapolation. The $\tau_{\text {had }} \tau_{\text {had }}$ channel uses templates built in a dedicated control region and the normalization is directly retrieved from the fit to data.

The $Z \rightarrow l l$ is a significant background for the $\tau_{\text {lep }} \tau_{\text {lep }}$ channel, while the top quark production affects both the $\tau_{\text {lep }} \tau_{\text {lep }}$ and the $\tau_{\text {lep }} \tau_{\text {had }}$ channels. The top-quark background refers to the production of $t \bar{t}$ pairs or to single top quarks. They are both estimated using simulations and CRs are defined in order to constrain their normalization. A $Z \rightarrow l l \mathrm{CR}$ in the $\tau_{\text {lep }} \tau_{\text {lep }}$ channel is used and defined, requiring the $m_{l l}$ to be $80<m_{l l}<100 \mathrm{GeV}$. Top CRs for both the $\tau_{\text {lep }} \tau_{\text {lep }}$ and $\tau_{\text {lep }} \tau_{\text {had }}$ channels are used and defined by inverting the $b$-jet veto requirement (in the $\tau_{\text {lep }} \tau_{\text {lep }}$ and $\tau_{\text {lep }} \tau_{\text {had }}$ selections, it is required to contain no $b$-tagged jets). 


\section{Statistical analysis}

A maximum likelihood fit is performed on data in order to extract the parameter of interest, namely the $\sigma_{H \rightarrow \tau \tau}$ total cross section. A fit model is constructed using the $m_{\tau \tau}^{\mathrm{MMC}}$ distributions in the SRs and only the event yields in the CRs. SRs are modeled by a product of Poisson distributions, each of this distribution representing the event yield in intervals of $m_{\tau \tau}^{\mathrm{MMC}}$ while CRs are modeled by a single Poisson distribution that describes the event count in that region. Systematic uncertainties are also taken into account as nuisance parameters and parametrized by Gaussian or log-normal distributions. Both theoretical and experimental uncertainties are considered. The impact of systematic uncertainties listed in decreasing order of their fractional impact on $\sigma_{H \rightarrow \tau \tau}$ is shown in Figure 2. The dominant systematic uncertainties are related to the signal theory uncertainties, particularly missing higher order QCD corrections for the ggF process, to the jet energy resolution and to the normalization of $Z \rightarrow \tau \tau$ and $Z \rightarrow l l$ backgrounds.

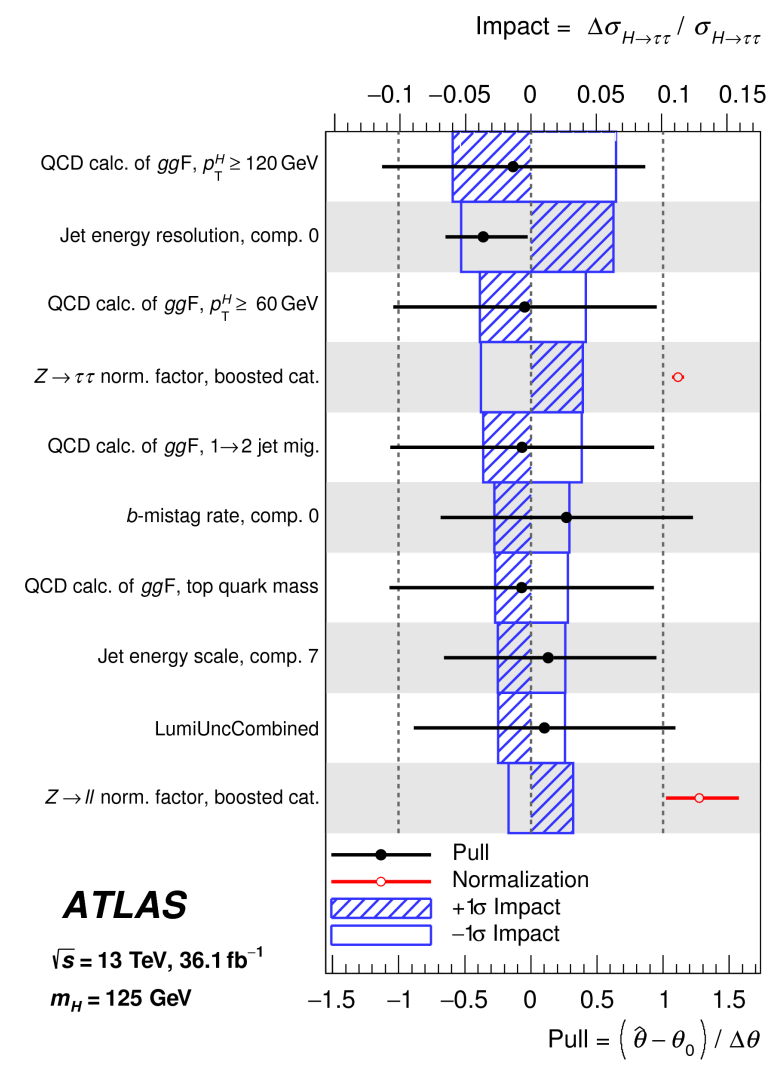

Figure 2: Fractional impact of systematic uncertainties on $\sigma_{H \rightarrow \tau \tau}$ as computed by the fit. The blue boxes show the impact of systematics while the filled circles show the deviations of nuisance parameters from their nominal values, normalized to their nominal uncertainty [4].

\section{Results}

The measured value of $\sigma_{H \rightarrow \tau \tau}$ is $3.77_{-0.59}^{+0.60}$ (stat.) ${ }_{-0.74}^{+0.87}$ (syst.) pb where all the relative contri- 


\begin{tabular}{lccr}
\hline Process & Particle-level selection & $\sigma[\mathrm{pb}]$ & $\sigma^{\mathrm{SM}}[\mathrm{pb}]$ \\
\hline$g g \mathrm{~F}$ & $N_{\text {jets }} \geq 1,60<p_{\mathrm{T}}^{H}<120 \mathrm{GeV},\left|y_{H}\right|<2.5$ & $1.79 \pm 0.53$ (stat.) \pm 0.74 (syst.) & $0.40 \pm 0.05$ \\
$g g \mathrm{~F}$ & $N_{\text {jets }} \geq 1, p_{\mathrm{T}}^{H}>120 \mathrm{GeV},\left|y_{H}\right|<2.5$ & $0.12 \pm 0.05$ (stat.) \pm 0.05 (syst.) & $0.14 \pm 0.03$ \\
VBF & $\quad\left|y_{H}\right|<2.5$ & $0.25 \pm 0.08$ (stat.) \pm 0.08 (syst.) & $0.22 \pm 0.01$ \\
\hline
\end{tabular}

Table 1: VBF and ggF cross section measurements in three exclusive regions. The definitions are based on the simplified template cross section framework [4].

butions of the Higgs production processes are assumed to be as predicted by the SM. This value is compatible with the SM prediction, which is $\sigma_{H \rightarrow \tau \tau}^{\mathrm{SM}}=3.46 \pm 0.13 \mathrm{pb}$, as shown in Figure 3 (a). In addition, since the VBF and boosted categories are sensitive to the VBF and ggF production mechanism respectively, a two parameter fit has also been performed to determine the cross sections of these production processes. The resulted values are $\sigma_{H \rightarrow \tau \tau}^{\mathrm{VBF}}=0.28 \pm 0.09$ (stat.) ${ }_{-0.09}^{+0.11}$ (syst.) pb and $\sigma_{H \rightarrow \tau \tau}^{g g F}=3.1 \pm 1.0$ (stat.) ${ }_{-1.3}^{+1.6}$ (syst.) pb. Both cross sections values are in agreement with the SM predictions (Figure $3(\mathrm{~b})$ ).

Furthermore, a first attempt to measure ggF and VBF cross sections using selections based on the simplified template cross section framework [8] has been performed. Table 1 shows the results using a three-parameter fit.

The observed (expected) significance of the signal excess relative to the background-only hypothesis is 4.4 (4.1) standard deviations. This result, combined with the Run 1 result [9] obtained using data collected at 7 and $8 \mathrm{TeV}$ center of mass energies, leads to an observed (expected) significance of 6.4 (5.4) standard deviations. This constitutes the first ATLAS observation of the $H \rightarrow \tau \tau$ decay.

\section{Conclusions}

Measurements of the Higgs production cross section in the $H \rightarrow \tau \tau$ decay channel have been presented. The analysis was performed using $36 \mathrm{fb}^{-1}$ of data collected during 2015 and 2016 by the ATLAS experiment at the LHC at a center of mass energy of $\sqrt{s}=13 \mathrm{TeV}$. An excess of events over the expected background was found with a significance of 4.4 (4.1) standard deviations. Combined with the Run 1 result, obtained using data collected at 7 and $8 \mathrm{TeV}$ center of mass energies, the observed (expected) significance is 6.4 (5.4) standard deviations, constituting the first $H \rightarrow \tau \tau$ ATLAS observation. The measured total cross section was found to be $\sigma_{H \rightarrow \tau \tau}=$ $3.77_{-0.59}^{+0.60}$ (stat.) ${ }_{-0.74}^{+0.87}$ (syst.) pb, in agreement with the SM expectation. In addition, measurements of the VBF and ggF production cross sections separately, and cross section measurements based on the simplified template cross section framework, have been performed, and they are all consistent with the SM predictions. 


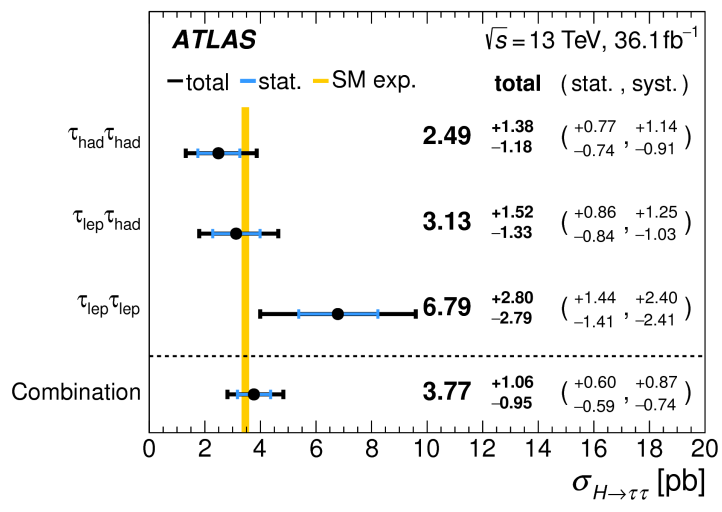

(a)

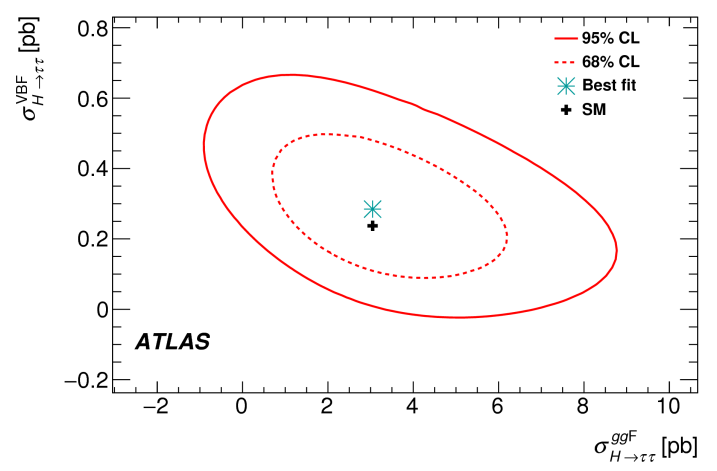

(b)

Figure 3: (a) $\sigma_{H \rightarrow \tau \tau}$ measurement in the various subchannels and for the combined result, with the SM prediction and its uncertainty shown in yellow, and (b) $95 \%$ and $68 \%$ C.L. contours in the plane of $\sigma_{H \rightarrow \tau \tau}^{V B F}, \sigma_{H \rightarrow \tau \tau}^{g g F}$ with the SM value indicated by the black point and the best-fit value shown as a star [4].

\section{References}

[1] ATLAS Collaboration, Phys. Lett. B 716(1):1-29 2012.

[2] CMS Collaboration, Phys. Lett. B 716:30-61 2012.

[3] ATLAS collaboration, JHEP 16082016045.

[4] ATLAS collaboration, Phys. Rev. D 990720012019.

[5] ATLAS Collaboration, JINST 3 S08003 2008.

[6] A. Elagin, P. Murat, A. Pranko, A. Safonov, Nucl. Instrum. Methods Phys. Res. A 6542011 481-489.

[7] T. Gleisberg et al., JHEP 022009007.

[8] D. de Florian et al. LHC Higgs Cross Section Working Group, CERN-2017-002-M, arXiv:1610.07922 [hep-ph].

[9] ATLAS Collaboration, JHEP 15042015117. 